of meteorology for whom the book seems to be intended. There are no references to original papers, and only one or two to text-books.

Mathematical proofs are kept few and short, and, as already hinted, historical experiments are not treated in great detail. As a rule all that is given is a short description in general terms, just enough to give the reader an idea of the observational basis of the subject: though a few important and recent experiments are a little more fully treated. Freed from such digressions, the story develops in a natural and logical fashion, and makes remarkably easy reading. The style is good, plain scientific English, which aims only at clarity and conciseness and relies on the subject-matter for its interest. Not even the chapter on rainmaking tempts the author to a lighter treatment, although few episodes in the history of science can be so rich in absurdity and chicanery.

The book is well illustrated with 29 very clear line drawings and 20 photographic plates. The latter include a short but well-chosen "cloud atlas" illustrating nine important cloud types, Prof. Mason's own remarkable photographs of artificially grown snow crystals, and some photographs of early cloud seeding trials which have not since been equalled for dramatic effect. This is a beautiful collection and adds greatly to the interest of the book.

There is a very adequate index for a book of its size. Printing and binding are excellent, in the usual style of the Cambridge University Press, and no misprints were noticed.

B. C. V. ODDIE tions which seems unnecessary. After all, ideas as abstract as those of geometry are taught at school. Minor algebraic results are derived for the probability-levels of $F$ although the validity of this distribution for analysing particular aspects of the variability of data is stated but not dis. cussed. Many practical examples are given with the arithmetic worked through in detail but they are nearly always very artificial. Either they deal with such subjects as coin tossing, balls in boxes, seating at table, etc., or, where framed in terms of observations made for a scientific purpose, the results have been constructed only as a numerical counterpart of the theory.

I do not think a natural or social scientist would obtain much of value from the algebraic proofs in this book. He could find from the text how to make estimates and apply tests of statistical significance given measurements suitable for such procedures. There would be little guidance on how he should determine what methods were useful and valid for analysing the data from a scientific investigation. He would spend his time more fruitfully studying a text which attempted to link an intuitive presentation of the principles of statistical theory with empirical studies of actual observations, using much arithmetic. There are a number of books of this kind in the tradition of Sir A. Bradford Hill's classic Principles of Medical Statistics. Only thus can the student hope to obtain some insight into the uses of the elegant mathematical methods in the interpretation of crude, unaccommodating, untidy observations.

W. BRASS

\section{STATISTICS FOR STUDENTS OF THE NATURAL AND SOCIAL SCIENCES}

Introduction to Probability and Statistics

By Henry L. Alder and Edward B. Roessler. (Series of Undergraduate Books in Mathematics.) Pp. xii +289. (San Francisco and London: W. H. Freeman and Company, 1962.) $32 s$.

THIS is the second edition of a text-book first published in 1960. The main change is the addition of two chapters, on the $F$ distribution and analysis of variance. The book is written to meet the needs of students in the natural and social sciences for a short course in probability and statistics at an elementary mathematical level; only arithmetic and simple algebra are required. The topics covered are standard and unenterprising, namely: the organization and analysis of data; elementary probability; standard probability distributions (binomial, normal); sampling theory including $t$, chi-square and $F$ distributions; regression and correlation; analysis of variance; index numbers; time series. A brief and unsatisfactory discussion of multiple-range tests, the one slight deviation from orthodoxy in contents, is given without much confidence in Chapter 17.

The subject of statistics is taught, nowadays, to students with such varied qualifications and needs that there is scope for text-books dealing with the same principles and techniques from different angles and levels of sophistication. The re-issue of the book reviewed indicates that it has been popular. It is clearly and carefully written with well-presented tables and diagrams and also excellently printed and produced. Nevertheless, I think the method of exposition is wrongly conceived.

The development of the theory is formal by means of definitions and elementary algebraic proofs. Inevitably however, because of the restriction on the mathematics, the definitions have to be partial and many important results accepted without proof. Thus sampling is everywhere taken to be from a finite population and the expecta. tion of a variable is treated only for the number of occurrences of an event in $n$ trials; this leaves a gap in the logic of the relation between sampling and probability distribu-

\section{MICROBIOLOGICAL STANDARDIZATION}

Proceedings of the 7th International Congress for Microbiological Standardization

London, August 28 to September 1, 1961. Edited by A. F. B. Standfast, D. G. Evans, and B. G. F. Weitz. (International Association of Microbiological Societies: Permanent Section for Microbiological Standardization.) Pp. vii + 556. (Edinburgh and London: E. and S. Livingstone, Ltd., 1962.) 130s. net.

THE bulk of this heavy 'paper-back' comprises some seventy papers grouped into fourteen sections under such headings as "Adventitious Agents in Vaccines Prepared from Living Tissues" and "Helminth Vaccines". The sections include from three papers under "The Application of Biological Standards and Reference Preparations in International and National Requirements for Biological Products" to eight under "BCG Vaccine" and "Staphylococcal Antigens and Antibodies". Fifteen of the papers are in French (without English summaries). Several are less than two pages in length and, in view of recent criticisms about 'over-production of scientific literature', some papers could well have been contracted or even omitted altogether, especially when "the details of these experiments have been published previously" as one contributor admits.

As a reference volume the task of locating a specific paper should be easy. In fact, there is no subject-index, and the page headings give only the contributor's name opposite an abbreviation of the section heading. Nowhere are the titles of the papers listed and the "Contents" could most profitably have been expanded to include these under their appropriate section headings. The short "Reports of Sessions" at the end are a partial remedy.

The papers emanate from fourteen different countries (including U.S.S.R., Yugoslavia, Hungary and Czechoslovakia) onabling a comparison of standardization procedures in different countries to be made. This is a truly international volume, but rather expensive.

L. B. QUeSNeI 